\title{
DEVELOPMENT OF VIRTUAL REALITY APPLICATION FOR CULTURAL HERITAGE VISUALIZATION FROM MULTI-SOURCE 3D DATA
}

\author{
K. Choromański, J. Łobodecki, K. Puchała, W. Ostrowski* \\ Warsaw University of Technology, Faculty of Geodesy and Cartography, \\ Pl. Politechniki 1, Warsaw, Poland - (kchor22, kuba.lobodecki, puchala1.karol, w.d.ostrowski)@ gmail.com
}

\section{Commission II}

KEY WORDS: Virtual Reality, Visualization, Cultural heritage, Multi-source data, Unreal Engine, Mesh generalization, Oculus Rift

\begin{abstract}
:
Immersive technologies like Virtual or Augmented Reality (VR/AR) are lately becoming more and more popular in wide range of scientific applications. These technologies provide the most immersive way to present spatial data such as point clouds or 3D models. This type of solutions also has significant potential for virtual presentation of cultural heritage. Combination of highquality photogrammetric 3D models, virtual reality technologies and an advanced visualization engine may bring effect in the form of a nearly real-world experience which may be very useful in terms of popularization as well as research in the area of cultural heritage. In this paper we would like to present results of experimental approach to establish VR system for the Museum of King John III's Palace at Wilanów in Warsaw, Poland.
\end{abstract}

\section{INTRODUCTION}

Nowadays, modern technologies are being used more and more often to facilitate access for cultural heritage. Popularity of online tours through so-called Virtual Tour is rising. Cultural heritage objects can be presented online by various methods, one of the fastest growing technologies that allows us to achieve this effect is Virtual Reality (VR). Virtual Reality is an artificially generated three-dimensional image, which through appropriate software and hardware, works on our senses (mainly sight and hearing) to imitate the reality surrounding us. Thanks to these features, this technology turns out to be extremely attractive to many institutions, including museums, where it was used since the beginning of $21^{\text {st }}$ century (Carrozzino \& Bergamasco, 2010). To make the experience of using VR more accessible to an ordinary recipient, often interactive elements are implemented in the virtual world through so-called "serious games" (Anderson, et al., 2010).

To guarantee greatest interest on the part of the viewer and best presentation of objects, it must be ensured that the models of these objects reflect the reality as best as possible. Many people have already made attempts to create a realistic model of a real object in the virtual world. In literature, many different approaches to this problem can be found. One of them is to model the object only using photographic images. When the object is photographed in its entirety, with a sufficient coverage between the photos, it is possible to recreate the orientation of images in relation to each other and then create point cloud describing $3 \mathrm{D}$ geometry of the object with dense image matching techniques. Level of detail and quality of the final model depends entirely on images resolution. The obtained results might be very detailed, but they will not be metric without proper reference from separate measurements. This method was for example used by Canciani et al. (2016) and by Barsanti, et al. (2015).

Another interesting approach is creating models using terrestrial laser scanning and digital photogrammetry. After the laser scans have been oriented, an oriented point cloud is made. In the next stage it is modelled using 3D CAD programs and textured (Kersten, et al., 2017a; and Kersten, et al., 2017b).

In our approach, the models were created using integrated data from terrestrial laser scanning and photographic images. The object of our work is Museum of King John III's Palace at Wilanów. It is a baroque palace with a garden foundation, founded by John III Sobieski, currently being one of the oldest museums in the country and repository of Poland's royal and artistic heritage.

\section{VISUALIZED CHAMBERS}

Models for 5 rooms from the palace complex were made: Big Hall, North and South Gallery, Quiet Room and Hall of Feasts. Each of these rooms is richly decorated with frescoes, reliefs and pilasters. As it was mentioned before the main source of data used for 3D modelling and visualization was provided by the Museum. Department of Documentation and Digitalization. Within Museum run the archive which gathers digital documentation of the Palace, among this data it's possible to find point clouds from laser scanning of interiors and exterior of Palace, images and various 3D models. Terrestrial laser scans were made for all chambers which are area of the study, most of them were also photographed, however only part of the images was collected for photogrammetric purposes (with decent overlap). The quality of data differed because it was acquired by different providers $\left(3^{\text {rd }}\right.$ party companies) at different times. Based on the data received from the museum, the differences before and after maintenance works were noticeable. For some of the rooms, pictures had to been taken.

\subsection{Big Hall}

Big Hall is one of the largest and most ornamented halls in Palace at Wilanów. The data received from the museum were used to

\footnotetext{
${ }^{*}$ Corresponding author
} 


\begin{tabular}{|c|c|c|c|c|c|}
\hline \multirow{2}{*}{ Chamber } & \multicolumn{2}{|c|}{ Laser scanning dataset } & \multicolumn{2}{|c|}{ Image dataset } & \multirow{2}{*}{ Interior decorations } \\
\hline & $\begin{array}{l}\text { Number } \\
\text { of points }\end{array}$ & $\begin{array}{c}\text { Coordinate } \\
\text { System }\end{array}$ & $\begin{array}{c}\text { Number } \\
\text { of photos }\end{array}$ & Resolution [px] & \\
\hline Big Hall & 314706339 & Local & 52 & $5616 \times 3744$ & bas-reliefs and columns \\
\hline North Gallery & 1011159033 & State & 398 & $5792 \times 8688$ & $\begin{array}{l}\text { frescoes on the walls and partly on ceiling, } \\
\text { carved ceiling }\end{array}$ \\
\hline South Gallery & 746918728 & State & 361 & $5792 \times 8688$ & $\begin{array}{l}\text { frescoes on the walls and partly on ceiling, } \\
\text { carved ceiling }\end{array}$ \\
\hline Quiet Room & 440452002 & Local & 56 & $4000 \times 6000$ & frescoes on the walls and on the ceiling \\
\hline Hall of Feasts & 781000900 & Local & 76 & $4000 \times 6000$ & $\begin{array}{l}\text { frescoes on the walls, richly decorated doors, } \\
\text { carved ceiling, plafond }\end{array}$ \\
\hline
\end{tabular}

Table 1. Data used to model the room with their description of the details.

develop the model of this room. Photos from the museum are of poor quality for Big Hall and the mutual coverage is very low, decreasing to $10 \%$ in some places. New pictures cannot be taken due to renovation works. During the previous conservation works, which were done between acquisitions of laser scanning and images, architectural and decorative fragments of the old décor were uncovered. This difference can also be observed on data from various periods.

\subsection{North and South Gallery}

The garden galleries connect the main body of the palace with side wings which were added in the $18^{\text {th }}$ century. Their ceilings are decorated with the frescoes (nowadays in different state of preservation) by Michelangelo Palloni. Some of the murals on the walls are also attributed to this artist. There was no need to take scans and photos because relevant data could be found in the museum's resources. Overlap of photos for the entire gallery was at the level of $70-80 \%$. In this part of the museum there is also the equestrian statue of John III Sobieski, which was also captured on scans and photos.

\subsection{Quiet Room}

Quiet Room is a chamber on the first floor of the Palace at Wilanów, located directly above the King's Bedroom. The walls and the ceiling of this low interior are covered with illusionistic mural paintings. As a result of conservation work, the missing fragments of the paintings were restored. The shape of the vault (oval) is unparalleled. Although it is the smallest room, it does not differ in detail from others. The museum's resources did not include photos of this room, that's why pictures had to be taken. Overlap is not satisfactory for the whole room, up to $50 \%$ in part of the room. It was not possible to make a larger number of photos later due to beginning of renovation works.

\subsection{Hall of Feasts}

Hall of Feasts is a room located on the last floor of the main body of the palace. The decor of the Hall of Feasts consists of varied decorations. Ceiling and facades are decorated with stucco (decorative elements made of gypsum or polyester resin). In the middle of the ceiling there is a plafond (oil painting on canvas). On all walls there are unique examples of eighteenth-century secular frescoes with figural representations. Due to insufficient number of photos in relation to the size of the room, in some places overlap was only $50 \%$.

\section{DATA PREPARATION STRATEGY}

As stated before data were acquired in various periods by different contactors. Due to that it was necessary to normalize and rearrange different types of measurements for each chamber. Point clouds for every object required merging as data were separated in .pts files. In two cases there was a need for changing real numbers separator in order to load clouds properly to CloudCompare (2018). After this process it was required to clear data from unnecessary elements such as noise or work equipment (e.g. scaffoldings). Final clouds were saved in .ply file format.

After preliminary preparations it was needed to combine data from photos and terrestrial laser scanning (TLS) to generate 3D meshes.

\subsection{Preparation of data from photos}

At first it was necessary to pre-process some of the data. For two chambers HDR images had to be joined manually from three separated photos. Also, after this operation, white balance process was carried out. Afterwards images were imported into Agisoft Photoscan (Agisoft, 2018) in order to place control points to bind point clouds from TLS to photos. 3D points were measured on point clouds with use of Limon Viewer. Than coordinates were placed on corresponding locations on images as markers in Agisoft Photoscan. In case of North and South Galleries photogrammetric targets were used as control points. For other chambers it was necessary to use natural points such as characteristic elements of murals.

\subsection{Preparation of data from laser scanning}

3.2.1 Scan merging and generalization: Laser scans from Museum archives were oriented and separated in parts by contractor. It was decided to merge them into one file in order to generate a mesh of whole room. Merged scans were too dense to use them for mesh generation because of limited efficiency of computers used for computation purposes. Merging and generalization were carried out with CloudCompare. Random method of cloud subsampling has been used. Sizes of clouds for each chamber were reduced to 90 million points as this value permits to carry out further computations in acceptable time and with preservation of high quality of final meshes. Mean distance between points in clouds was one to three millimetres after process of subsampling. However, further generalization still had to be carried out, after the meshes were created, in order to ensure fluent functioning of visualization. 


\begin{tabular}{c|c|c|c|c} 
Approach & Surface model & $\begin{array}{c}\text { Neighbour search } \\
\text { radius (milimeters) }\end{array}$ & Orientation method & $\begin{array}{c}\text { Time of computing } \\
\text { (seconds) }\end{array}$ \\
\hline A & Plane & $\begin{array}{c}9.651 \\
\text { (Default value) } \\
9.651 \\
\text { (Default value) }\end{array}$ & Barycenter & 69,27 \\
B & Plane & 8.000 & Binimum Spanning Tree & 65,23 \\
C & Plane & - & Barycenter & 13,36 \\
D & Triangulation & &
\end{tabular}

Table 2. Results of comparison of normal computing approaches. Part of North Gallery was chosen as test object (approx. 20 million points)

3.2.2 Computation of normal vectors: Surface reconstruction algorithm (structure from motion) used in Agisoft Photoscan use normal vectors of points to properly generate $3 \mathrm{D}$ mesh. Therefore, it was necessary to compute these values. CloudCompare provides a bunch of methods of normal vectors generating. In order to discover the best approach for described clouds the most promising methods were tested. Results of best fit plane and 2D triangulation were compared along with different values of neighbours' search radius and orientations methods (Fig. 1.). Outcomes of conducted research were presented in Table 2. Basing on results of test normal computations it was decided to use plane method with default value of neighbour search radius using Minimum Spanning Tree for orientation of normal vectors. This approach gives proper values even for complicated structures in acceptable time. Use of barycentre of object for normal vectors orientation works well for chambers with simple architecture on square plan. Also use of less than default search radius lead to errors in created meshes. 2D triangulation method turned out to be insufficient in terms of results of normal vector computation for point clouds of rooms.
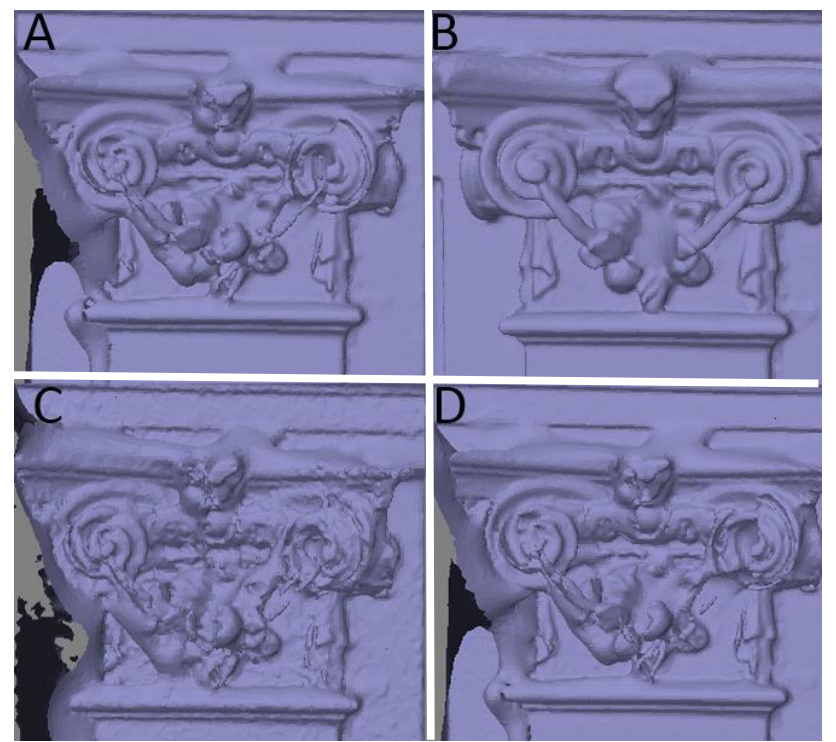

Figure 1. Meshes generated from clouds with differently computed normal vectors (particular methods are described in table 2.)

\section{MESH GENERATING AND GENERALIZATION WORKFLOW}

After first step of data preparation, subsampled point clouds with calculated normal vectors were used to crate $3 \mathrm{D}$ meshes with texture form oriented images. Next 3D meshes geometry have to simplified because of efficiency of final visualization. Two alterative workflows of these processes was proposed and tested.

\subsection{Data combining}

The fusion of colour information from photos and geometry from TLS was conducted in Agisoft Photoscan. Pre-processed clouds were imported to Photoscan project with images already loaded, aligned and oriented in TLS point cloud reference system. Afterwards meshes were generated on high detail level along with textures.

Various algorithms of texture mapping were tested. Generic and adaptive orthophoto modes were considered in process. As one can see on Figure 2. second one is easier to interpret by human which may be useful when it would come to necessity of manual texture correction. However, textures mapped in adaptive orthophoto mode were significantly deformed when used in final visualization (figure 3 .) what can be related with texture mapping algorithm implemented in Unreal Engine.
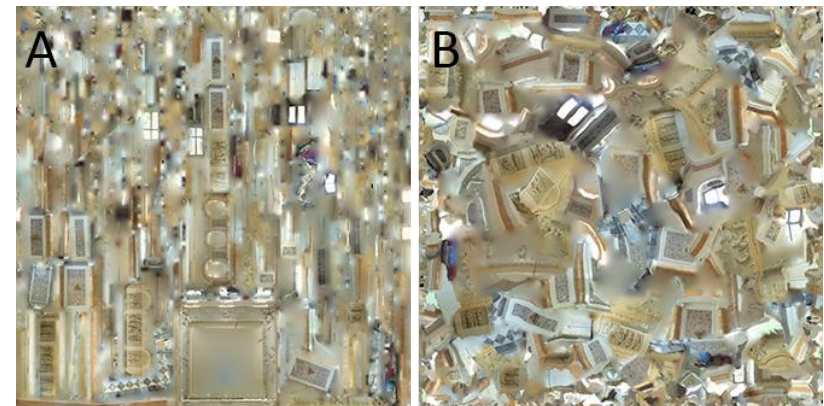

Figure 2. Textures in adaptive orthophoto (A) and generic (B) mapping mode.
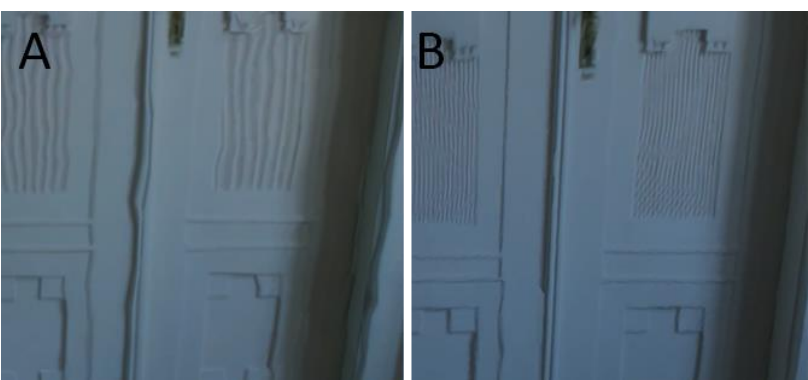

Figure 3. Comparison of adaptive orthophoto (A) and generic (B) textures mapped on mesh in Unreal Engine.

Another important parameter for visualization quality was texture resolution. In order to achieve photorealistic effect, it was necessary to achieve resolution close to original photos. Maximum resolution that could be used in Unreal Engine is $8192 \times 8192$, so such textures were generated. In case of bigger chambers: Hall of Feasts, North and South Galleries two textures in separate files for one mesh were used. Exact information about texture generating for each room are described in table 3. 


\begin{tabular}{c|c|c|c} 
Chamber & $\begin{array}{c}\text { Surface } \\
{\left[\mathbf{m}^{2}\right]}\end{array}$ & Texture size & $\begin{array}{c}\text { Avg. GSD of } \\
\text { texture }[\mathbf{m m}]\end{array}$ \\
\hline Big Hall & 396.4 & $8192 \times 8192$ & 2.4 \\
North Gallery & 404.6 & $2 \times 8192 \times 8192$ & 1.7 \\
South Gallery & 518.3 & $2 \times 8192 \times 8192$ & 2.0 \\
Quiet Room & 178.9 & $8192 \times 8192$ & 1.6 \\
Hall of Feasts & 397.9 & $2 \times 8192 \times 8192$ & 1.7
\end{tabular}

Table 3. Area of each chamber compared with texture size and GSD of final textures.

\subsection{Data processing for use in game engine}

In order to prepare data for game engine there was a need for model generalization and generation of additional $2 \mathrm{D}$ textures (in PNG format). There are numerous ways to achieve this goal. Two workflows of mesh and texture adaptation for Unreal game engine were developed.

In the first approach (Fig. 4. A) models exported from Photoscan were generalized in Instant Meshes (Jacob, et al. 2015) opensource software. This solution provides algorithms for mesh generalization to given number of vertices. In this process information about UV coordinates of reduced mesh is being loosed. Afterwards simplified mesh was imported into Blender (Blender Foundation, 2018) 3D manipulation program which gives a possibility to bake texture UV coordinates from original high-poly mesh to generalized one. Moreover, Blender comes with function of normal and specular map generation. Normal (Bump) maps are a special type of texture which stores information about localization of texture element in space what could store high level of details on mesh without extending its real vertex count while specular map represents level of reflectivity for given mesh area. Finally, low-poly mesh with baked UV coordinates is exported as FBX file that is readable for Unreal Engine.

Second approach (Fig. 4. B) assumed use of Photoscan feature of mesh decimating to decrease model vertex count. Afterwards texture was generated on already generalized model by Build Texture function in the same software so, in this solution, there is no need for additional UV coordinates baking. Unfortunately, Photoscan is not able to generate specular and normal textures so another solution had to be proposed. Open-source software called Materialize (Bounding Box Software, 2018) provides such features along with wide range of texture improvements and adjustments. Finally, the mesh is imported into Blender in order to adjust its position and scale to use it in game engine.

Both approaches gave similar results, however second one was much more convenient and quicker - there is no need of import original quality mesh into Blender, which proved to be quite problematic when it comes to work with very dense model. Moreover, Materialize offers wider range of texture adjustment possibilities than Blender.

\section{GAME ENGINE}

Game engine is a software which simplifies video games (or game similar product like animation, visualization, simulation) production. The main purpose of game engine is to provide as many ready to use program pieces as possible, so all developer attention can be given to content creation. As a result, these are
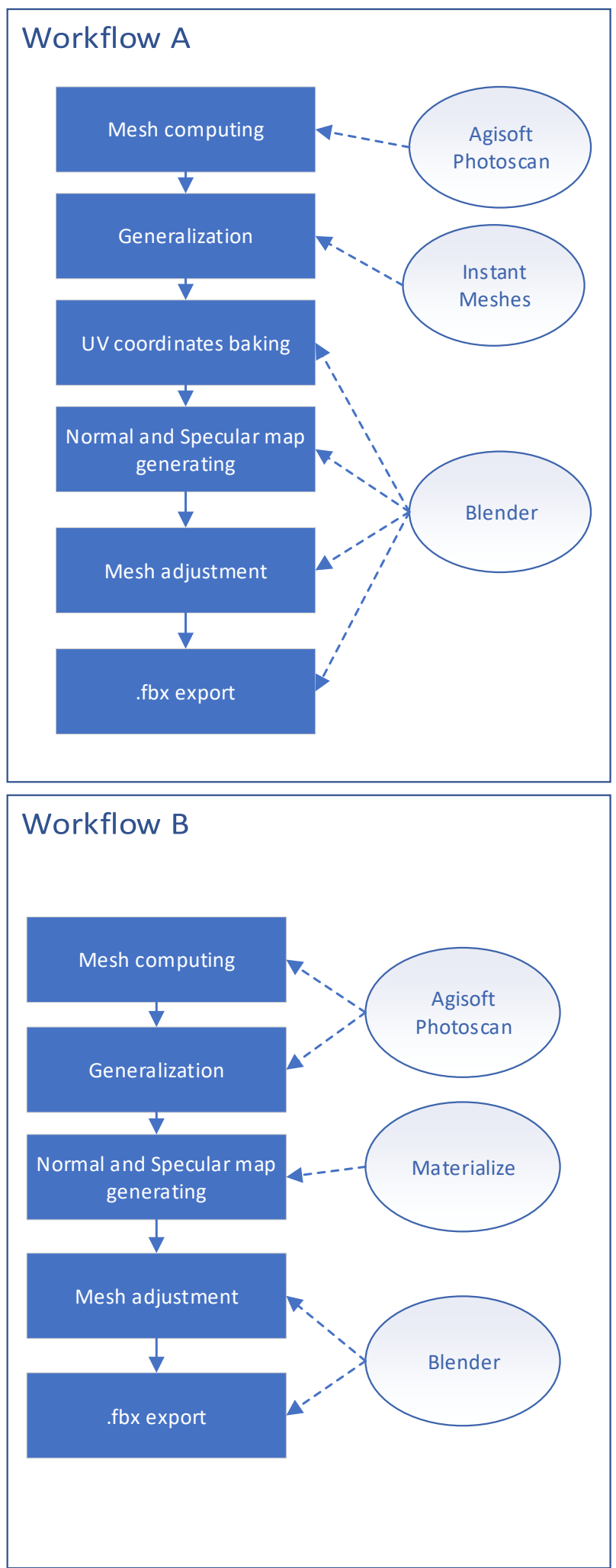

Figure 4. Comparison of data preparation workflows.

very extensive software allowing you to i.a. work in $2 \mathrm{D}$ and $3 \mathrm{D}$ environment, graphic rendering (models, materials, lighting and shading), events, control, user interface, sound handling and finally managing a virtual reality headset. Overwhelming 
majority of present-day serious game productions is maintained by one of available game engines.

There is a plenty of game engines available on the market. Almost all of them are distributed with a license which allows us to use it free of charge until the income threshold is reached. Based on a survey made by Statista web portal in 2014 the three most popular engines (intended for 3D production) were: Unity by Unity Technologies, Unreal Engine by Epic Games and CryEngine by Crytek (Statista, 2014).

All three cover most of the necessary functionalities (such as 3D graphics rendering, realistic lighting, VR support). For purposes of implementing VR for Palace at Wilanów Unreal Engine was chosen because of two main reasons: First is the relatively best graphic quality (in terms of closeness to the real world) supplied out of the box. Second is the visual scripting system called "Blueprints". It allowed team members who were not programmers to get involved in the process of game (VR) creation.

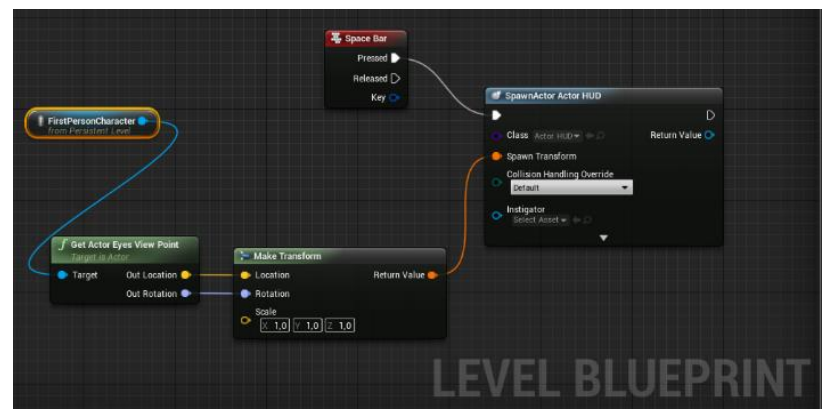

Figure 5. Sample blueprint responsible for showing user interface element on space bar key pressing action.

\section{OCULUS RIFT VR SYSTEM}

It was decided to use the Oculus Rift Virtual Reality system, which is one of the most popular on the market. It contains googles, two controllers and two sensors with which it is possible to place user in three-dimensional space. Googles tracks head movement and direction so on each eye screen proper image from virtual world is displayed.

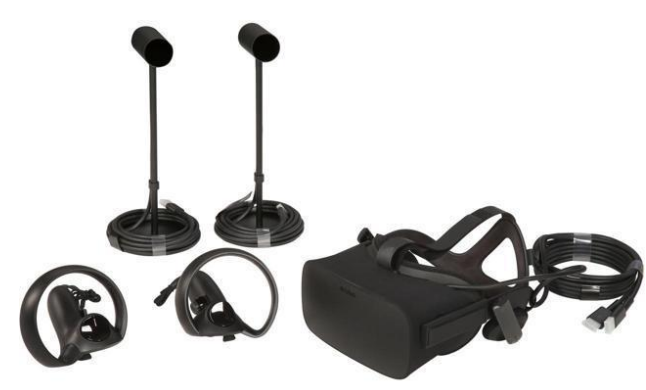

Figure 6. Content of Oculus Rift VR headset.

It is important to mention that due to the necessity to render display two independent (stereoscopic) images in stable framerate of $90 \mathrm{fps}$ (frames per second), powerful hardware is required, and additional performance optimizations has to be done. For testing a PC was used equipped with Intel i5-4590 processor, NVidia RTX 2070 graphic card and 16GB of RAM. Maintaining an optimal level of smoothness and following VR design principles is extremely important because of the risk of virtual reality sickness (Hyun et al., 2018). That can be caused by drops of presented frames per second, dynamic acceleration or field of view change and head movement uncontrolled by the user. It is the reason for selecting a mobility system based on teleportation.

\section{IMPLEMENTATION OF VISUALIZATION}

Last part of presented work was to combine created 3D models with additional information, scripts, lights and implement all these elements in VR system.

\subsection{Models arrangement}

The experiment involved modelling five rooms due to restrictions on the museum's side. Therefore, a problem has occurred with location in space of every chamber. It has been resolved by placing virtual walls corresponding to building plan which can be seen at figure 7 . Thanks to that user gets additional information about a place in the palace where the chambers are located.

Moreover, special collider objects have to been placed to make it impossible to go through the walls.

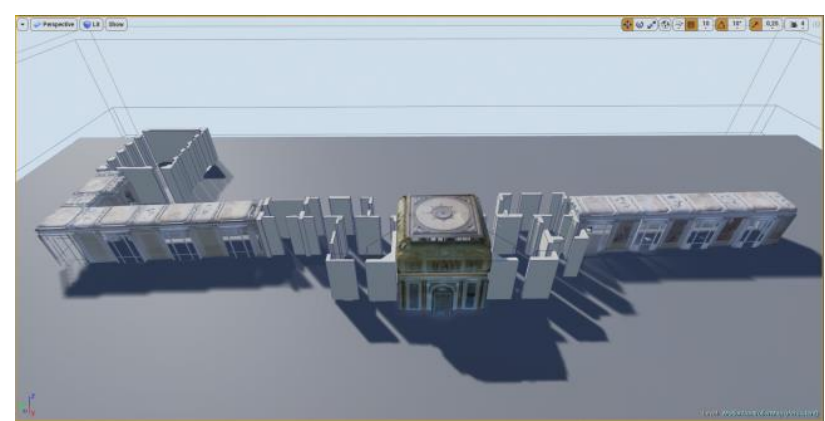

Figure 7. Overhead projection of the palace ground floor, where only three chambers were modelled with textured meshes (Both Galleries and Big Hall). Other chambers were placed in 3D as extrusion of walls outlines.

\subsection{Lighting}

To get the most realistic looking and immersive visualization the adequate illumination of the models is essential. Firstly, the main light which purpose is to imitate the sun light was placed on the scene. With this approach main light has provided strong rays falling into chambers through windows. This would be sufficient if the ray tracing technology was used. First use of it in real-time rendering was tested by Ludvigsen and Elster (2010) but its practical application will be possible in near feature thanks to new Nvidia RTX system (Nvidia, 2018). For now, the lighting reflection is not possible due to technical limitations so additional supportive lights are necessary. Their purpose is to simulate the natural balanced illumination of the entire room.

Visualization of the Palace at Wilanów has been illuminated by sky light and directional light source which imitates the sun. Additionally, for each chamber three point lights were placed to provide balanced illumination. The number of three lights is caused by the technical restriction imposed by the Unreal Engine.

\subsection{Historical information presentation}

Due to educational character of the project, it is crucial to provide the possibility of displaying information about the objects found in the museum. To achieve that, special HUD (Head-Up Display) is presented after pointing on unique object as shown at figure 9 . In addition, after the first entry into the chamber a dedicated sound record is played with the main historical facts about it. Sound record is also embedded in 3D space which makes it 
possible to hear surround audio. The presented information comes from the extensive database of objects maintained by the museum.

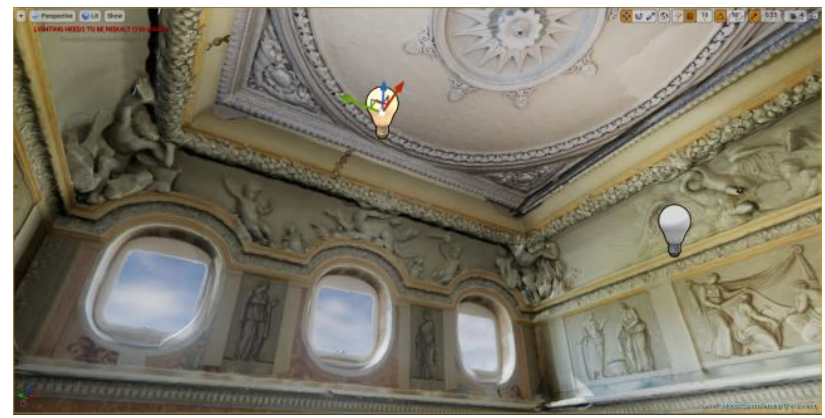

Figure 8. Example of additional lighting placement inside Big Hall chamber.

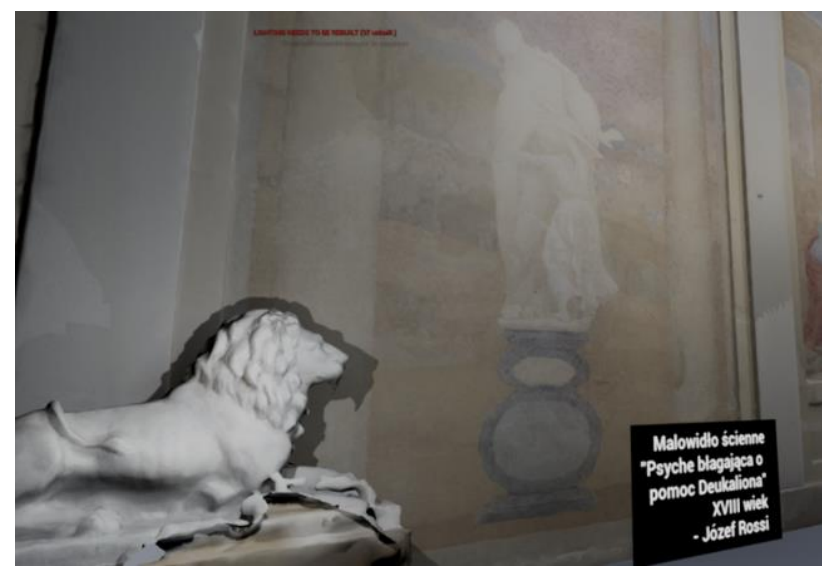

Figure 9. Example of final visualization (North Gallery), on the right-side HUD with additional information about wall painting is visible.

\subsection{Virtual reality systems}

To implement the virtual reality feature mostly Unreal Engine built-in functions were used. Default VR pawn (player representation in virtual world) was used with pre-implemented teleportation movement system. To the embedded player hands, we add a possibility to point at specific object and see the board with additional information.

\section{CONCLUSIONS}

This contribution described the process of implementation of virtual reality visualization basing on multi-source 3D data from archives of Museum of King John III's Palace at Wilanów for Oculus Rift VR system. Such visualization makes it possible to remotely familiarise Museum architecture and history in the closest way to a real visit. Such product may be an important contribution to Museum's promotion and a very useful and effective educational material because it is possible to share information about history of the Palace directly through the visualization.

Additionally, use of Unreal Engine gives nearly no restrictions on further expanding and modifying final product by implementing new functionalities and adding other Palace chambers which may increase its educational value.

Chosen game engine - Unreal Engine proved to be very effective and convenient as a tool for creating such visualizations.
However, the whole process of model preparation from multisource data is quite laborious and demanding in the matter of required computing power. Also, manual amendments were needed in order to achieve satisfactory final effect. Basing on obtained results and conducted tests it would be even more problematic if only photos were to be used in process. Large number of problems during mesh generation process were related to the fact that the data obtained from Museum's archives were gathered by numerous contractors for different goals. Final effect would be more consistent and easier to obtain if appropriate measurements were carried out specifically for this purpose.

Proper computation of normal vectors is required in order to receive proper mesh with mesh generation algorithm used in Agisoft Photoscan. CloudCompare normal computation algorithms are fully sufficient to achieve this goal. Minimum Spanning Tree orientation method proved to give the best results for discussed objects.

Due to the computing power of modern computers and limitations of game engines it is impossible to use full-quality data from photos and TLS measurements directly for visualization purposes. Generalization of generated mesh is therefore indispensable. Using the second of proposed workflows gave better results in terms of time consumption.

Many photos were used to create textures for the models. Their quality and coverage had a significant impact on the final effect. As a result, textures with a resolution of 500 pixels per metre (2 mm GSD) were sufficient.

Moreover, use of virtual reality systems requires a decent computer device in order to work smoothly, which limits the possibility of wide use of visualization. Nevertheless, experience of interacting with VR visualization is very immersive and it would be impossible to reproduce it without such system.

\section{ACKNOWLEDGEMENTS}

The authors acknowledge support of students form Warsaw University of Technology Geodesy and Cartography Student's Club in model generating and game engine implementation. Museum of King John III's Palace at Wilanów in Warsaw, Poland help by sharing data and consultations is gratefully acknowledged.

\section{REFERENCES}

Anderson, E. F., McLoughlin, L., Liarokapis, F., Peters, C., Petridis, P., \& De Freitas, S. 2010. Developing serious games for cultural heritage: a state-of-the-art review. Virtual reality, 14(4), 255-275.

Agisoft, 2018. Agisoft Photoscan Professional Edition Software, Version 1.4.4. http://www.agisoft.com (20 December 2018)

Blender Foundation, 2018. Blender Software, Version 2.79b. https://www.blender.org (20 December 2018)

Bounding Box Software, 2018. Materialize Software, Version 1.78. http://boundingboxsoftware.com/materialize/ (20 December 2018)

Canciani, M., Conigliaro, E., Grasso, M. D., Papalini, P., \& Saccone, M. 2016. 3D survey and augmented reality for cultural heritage. The case study of aurelian wall at castra praetoria in 
rome. International Archives of the Photogrammetry, Remote Sensing \& Spatial Information Sciences, 41.

Carrozzino, M., \& Bergamasco, M. 2010. Beyond virtual museums: Experiencing immersive virtual reality in real museums. Journal of Cultural Heritage, 11(4), 452-458.

CloudCompare, 2018. CloudCompare Software, Version 2.9.1 Omnia. https://www.danielgm.net/cc/ (20 December 2018)

Jakob, W., Tarini, M., Panozzo, D., \& Sorkine-Hornung, O. 2015. Instant field-aligned meshes. ACM Trans. Graph., 34(6), pp. 189:1-189:15. https://github.com/wjakob/instant-meshes

Kersten, T. P., Tschirschwitz, F., \& Deggim, S. 2017a. Development of a virtual museum including a $4 D$ presentation of building history in virtual reality. The International Archives of Photogrammetry, Remote Sensing and Spatial Information Sciences, 42, 361 .

Kersten, T. P., Büyüksalih, G., Tschirschwitz, F., Kan, T., Deggim, S., Kaya, Y., \& Baskaraca, A. P. 2017b. The selimiye mosque of edirne, turkey-an immersive and interactive virtual reality experience using htc vive. International Archives of the Photogrammetry, Remote Sensing \& Spatial Information Sciences, 42.

Krishnamurthy, V., \& Levoy, M. 1996. Fitting smooth surfaces to dense polygon meshes. In Proceedings of the 23rd annual conference on Computer graphics and interactive techniques $\mathrm{pp}$. 313-324.

Kim, H. K., Park, J., Choi, Y., \& Choe, M. 2018. Virtual reality sickness questionnaire (VRSQ): Motion sickness measurement index in a virtual reality environment. Applied ergonomics, 69, 66-73.

Ludvigsen, H., \& Elster, A. C., 2010. Real-Time Ray Tracing Using Nvidia OptiX. In Eurographics (Short Papers) pp. 65-68.

Nvidia, 2018. Official Nvidia RTX webpage. https://developer.nvidia.com/rtx/raytracing (20 December 2018)

Statisa, 2014. Leading game engines used by video game developers in the United Kingdom (UK) 2014.

https://www.statista.com/statistics/321059/game-engines-usedby-video-game-developers-uk/ 